\title{
Modulation Instability and Its Impact in Multispan Optical Amplified IMDD Systems: Theory and Experiments
}

\author{
Rongqing Hui, Maurice O'Sullivan, Alan Robinson, \\ and Mike Taylor
}

\begin{abstract}
The nonlinear interaction between amplified spontaneous emission noise and copropagating signal in a dispersive optical fiber is investigated both theoretically and experimentally. A transfer matrix formulation is used to solve the nonlinear propagation equation. A continuous wave $(\mathrm{CW})$ input optical signal format isolates the effect of modulation instability (MI) from the signal self-phase modulation. The change of the relative intensity noise (RIN) spectrum at an optical receiver caused by MI has been analyzed extensively in multispan optical amplified systems, and thus the impact of MI in the performance of intensity modulation and direct detection (IMDD) systems is evaluated. Performance of systems with distributed dispersion compensation (DC) and a lumped DC at the receiver are compared in terms of the effect of MI, the results show that the highest efficiency of DC to reduce the effect of MI can be achieved by putting a lumped DC module at the receiver side. Excellent agreement between theory and experiment demonstrates a good understanding of the mechanism of MI and its impact in the performance of terrestrial optical transmission systems.
\end{abstract}

Index Terms-Fiber nonlinearity, modulation instability, optical communications.

\section{INTRODUCTION}

$\mathbf{N}$ ONLINEAR effects have been reported to be significant in optical fiber transmission systems [1]-[2]. Of particular interest is the Kerr effect where the refractive index of the fiber medium strongly depends on the intensity of the optical signal. In a nonsoliton system the Kerr effect broadens the signal optical spectrum through self-phase modulation (SPM), this broadened spectrum is mediated by fiber dispersion and causes a performance degradation. In addition, four wavemixing (FWM) between the signal and amplified spontaneous emission (ASE) noise has been reported to cause performance degradations, in systems using in-line optical amplifiers [3]-[6]. This later effect is commonly referred to as modulation instability (MI).

The mechanism for system performance degradation caused by MI depends on system type. In coherent transmission systems, degradation is mainly caused by the broadening of the optical spectrum [7]. On the other hand, for an

Manuscript received September 27, 1996; revised March 26, 1997.

R. Hui and M. O'Sullivan are with Nortel, Broadband Networks, Ottawa, Ont. K1Y 4H7, Canada.

A. Robinson and M. Taylor are with Nortel Technology, London Road, Harlow, Essex, CM17 9NA, England.

Publisher Item Identifier S 0733-8724(97)05152-9. intensity-modulation direct-detection (IMDD) optical system, the spreading of the optical spectrum must be accompanied by dispersion mediated phase to intensity conversion to produce an increase in the relative intensity noise (RIN) within the receiver baseband. For IMDD systems with zero fiber dispersion, FWM between the signal and ASE has been studied both numerically and analytically [3]-[4], [8]-[9], it has been shown that, the broadening of the signal optical spectrum caused by MI does not degrade bit error rate. This is so since, as long as the system optical bandwidth is wide enough to accommodate the broadened spectrum, no phase to amplitude conversion will take place. In most of the practical cases, however, fiber dispersion is locally non zero. Under these circumstance, phase noise caused by MI can be converted into intensity noise via dispersion. The analytical approach used in [8]-[9] fails to describe this wide category of practical systems.

In this paper, we present a semi-analytical model to calculate the effect of MI in optical amplified multispan IMDD systems with nonzero fiber dispersion. Experiments are conducted to verify the theoretical results. We will also show that the system penalty due to MI can be reduced by dispersion compensation (DC). The optimum amount of DC and its optimum position in terms of reducing MI effect are discussed.

\section{THEORETICAL ANALYSIS}

\section{A. Transmission Matrix Formulation}

Wave propagation along a lossy, single-mode and linearly polarized fiber can be described by the nonlinear Schrodinger equation [1]

$$
\begin{aligned}
\frac{\partial A(z, t)}{\partial z}= & \frac{-i}{2} \beta^{\prime \prime} \frac{\partial^{2} A(z, t)}{\partial t^{2}}+i \gamma|A(z, t)|^{2} A(z, t) \\
& -\frac{\alpha}{2} A(z, t)
\end{aligned}
$$

where $A(z, t)$ is the electrical field, $\gamma=\omega_{0} n_{2} / c A_{\text {eff }}$ is the nonlinear coefficient of the fiber, $\omega_{0}$ is the angular frequency, $n_{2}$ is the refractive index of the fiber, $c$ is the speed of light, $A_{\mathrm{eff}}$ is the effective fiber core area, $\beta^{\prime \prime}$ is the fiber dispersion parameter, and $\alpha$ is the fiber attenuation. High-order dispersions have been ignored here. 
The steady-state solution of (1) is $z$-dependent

$$
A_{0}(z)=\sqrt{P_{0}} \exp \left(i \gamma\left|A_{0}(z)\right|^{2} z\right) \exp \left(-\frac{\alpha}{2} z\right)
$$

and the signal optical power can vary for tens of decibels along the fiber in practical systems because of the attenuation. Therefore, a simple mean-field approximation over the transmission fiber is usually not accurate enough. For an easy solution, the fiber can be divided into short sections and a mean-field approximation can be applied within each section. For example, in the $j$ th section with length $\Delta z_{j}$, (1) becomes

$$
\frac{\partial A_{j}(z, t)}{\partial z}=\frac{-i}{z} \beta^{\prime \prime} \frac{\partial^{2} A_{j}(z, t)}{\partial t^{2}}+i \gamma_{j}\left|A_{j}(z, t)\right|^{2} A_{j}(z, t)
$$

where

$$
\gamma_{j}=\frac{1-\exp \left(-\alpha \Delta z_{j}\right)}{\alpha \Delta z_{j}} \gamma
$$

With the assumption that noise power presence at the input of the fiber is much smaller than the signal power, the solution of (3) can be written as

$$
A_{j}(z, t)=\left[A_{0 j}+\tilde{a}_{j}(z, t)\right] \exp \left(i \gamma_{j}\left|A_{0 j}\right|^{2} z\right)
$$

where $A_{0 j}$ is the steady-state solution of (3), $\tilde{a}_{j}(z, t)$ is a small perturbation and $\tilde{a}_{j}(z, t) \ll A_{0 j}$ is assumed. With linear approximation of the noise term

$$
\begin{aligned}
\frac{\partial \tilde{a}_{j}(z, t)}{\partial z}= & \frac{-i}{2} \beta^{\prime \prime} \frac{\partial^{2} \tilde{a}_{j}(z, t)}{\partial t^{2}} \\
& +i \gamma_{j}\left[\left|A_{0 j}\right|^{2} \tilde{a}_{j}(z, t)+A_{0 j}^{2} \tilde{a}_{j}(z, t)\right] .
\end{aligned}
$$

Higher order terms of $\tilde{a}_{j}(z, t)$ have been neglected and the symbol $*$ denotes complex conjugate. We need to emphasize that the linear equation for the perturbation shown in (6) is valid only when the perturbation is small and as a result of this approximation, the pump depletion is not taken into account.

By Fourier transformation of (6) into frequency domain [10]

$$
\begin{aligned}
\frac{\partial a_{j}(\omega, z)}{\partial z}= & \frac{i}{2} \omega^{2} \beta^{\prime \prime} a_{j}(\omega, z)+i \gamma_{j}\left|A_{0 j}\right|^{2} a_{j}(\omega, z) \\
& +i \gamma_{j} A_{0 j}^{2} a_{j}^{*}(-\omega, z) \\
\frac{\partial a_{j}^{*}(-\omega, z)}{\partial z}= & \frac{-i}{2} \omega^{2} \beta^{\prime \prime} a_{j}^{*}(-\omega, z)-i \gamma_{j}\left|A_{0}\right|^{2} a_{j}^{*}(-\omega, z) \\
& -i \gamma_{j} A_{0 j}^{*^{2}} a_{j}(\omega, z) .
\end{aligned}
$$

The formal solution of linear differential (7)-(8) can be expressed in a matrix format

$$
\left[\begin{array}{c}
a_{j+1}\left(\omega, z_{j}+\Delta z_{j}\right) \\
a_{j+1}^{*}\left(-\omega, z_{j}+\Delta z_{j}\right)
\end{array}\right]=\left[\begin{array}{cc}
M_{11}^{(j)} & M_{12}^{(j)} \\
M_{21}^{(j)} & M_{22}^{(j)}
\end{array}\right]\left[\begin{array}{c}
a_{j}\left(\omega, z_{j}\right) \\
a_{j}^{*}\left(-\omega, z_{j}\right)
\end{array}\right] .
$$

When we take into account the linear attenuation of the signal, from the start of one section to the start of the next section, we have

$$
\begin{aligned}
{\left[\begin{array}{c}
a_{j+1}\left(\omega, z_{j}+\Delta z_{j}\right) \\
a_{j+1}^{*}\left(-\omega, z_{j}+\Delta z_{j}\right)
\end{array}\right]=} & {\left[\begin{array}{ll}
M_{11}^{(j)} & M_{12}^{(j)} \\
M_{21}^{(j)} & M_{22}^{(j)}
\end{array}\right]\left[\begin{array}{c}
a_{j}\left(\omega, z_{j}\right) \\
a_{j}^{*}\left(-\omega, z_{j}\right)
\end{array}\right] } \\
& \cdot \exp \left(-\frac{\alpha}{2} \Delta z_{j}\right)
\end{aligned}
$$

where $\Delta z_{j}=z_{j+1}-z_{j}$ and

$$
\begin{aligned}
M_{11}^{(j)} & =\frac{e^{i k_{j} z}-r_{f j} r_{b j} e^{-i k_{j} z}}{\left|1-r_{f j} r_{b j}\right|} \\
M_{12}^{(j)} & =\frac{r_{b j}\left(e^{-i k_{j} z}-e^{i k_{j} z}\right)}{\left|1-r_{f j} r_{b j}\right|} \\
M_{21}^{(j)} & =\frac{r_{f j}\left(e^{i k_{j} z}-e^{-i k_{j} z}\right)}{\left|1-r_{f j} r_{b j}\right|} \\
M_{22}^{(j)} & =\frac{e^{-i k_{j} z}-r_{f j} r_{b j} e^{i k_{j} z}}{\left|1-r_{f j} r_{b j}\right|} \\
r_{f j} & =\frac{k_{j}-\beta \omega^{2}-\gamma_{j}\left|A_{0 j}\right|^{2}}{\gamma_{j} A_{0 j}^{2}}=\frac{-\gamma_{j} A_{0 j}^{*^{2}}}{k_{j}+\beta \omega^{2}+\gamma_{j}\left|A_{0 j}\right|^{2}} \\
r_{b j} & =\frac{k_{j}-\beta \omega^{2}-\gamma_{j}\left|A_{0 j}\right|^{2}}{\gamma_{j} A_{0 j}^{* 2}}=\frac{-\gamma_{j} A_{0}^{2}}{k_{j}+\beta \omega^{2}+\gamma_{j}\left|A_{0 j}\right|^{2}} .
\end{aligned}
$$

Equations (7)-(8) have two eigenmodes whose propagation constants are equal in magnitude and opposite in sign, given by

$$
k_{j}= \pm \sqrt{\left(\beta \omega^{2}+\gamma_{j}\left|A_{0 j}\right|^{2}\right)^{2}-\left(\gamma_{j}\left|A_{0 j}\right|^{2}\right)^{2}}
$$

Here, $r_{f j}$ and $r_{b j}$ are the effective reflectivity for the two eigenmodes respectively, therefore, the sign of $k$ should be chosen such that $\left|r_{f j}\right| \leq 1$ and $\left|r_{b j}\right| \leq 1 . \beta=\beta^{\prime \prime} / 2$ was used in the above equations for simplicity of notation.

The evolution of the noise along the fiber can then be calculated simply by matrix multiplication

$$
\left[\begin{array}{c}
a(\omega, L) \\
a^{*}(-\omega, L)
\end{array}\right]=\left[\begin{array}{ll}
B_{11} & B_{12} \\
B_{21} & B_{22}
\end{array}\right]\left[\begin{array}{c}
a(\omega, 0) \\
a^{*}(-\omega, 0)
\end{array}\right] \exp \left(-\frac{\alpha}{2} L\right)
$$

with

$$
\left[\begin{array}{ll}
B_{11} & B_{12} \\
B_{21} & B_{22}
\end{array}\right]=\prod_{j=1}^{N}\left[\begin{array}{ll}
M_{11}^{(j)} & M_{12}^{(j)} \\
M_{21}^{(j)} & M_{22}^{(j)}
\end{array}\right]
$$

and where $L$ is the fiber length and $N$ is the total number of sections.

\section{B. Power Spectrum of the Optical Field}

According to the Wiener-Khintchine theorem, the power spectrum of the optical field is propotional to the square of the modulus of the Fourier transformation of the complex field amplitude. If the field is sampled over a time interval $T$

$$
S_{t}(\omega, z)=\lim _{T \rightarrow \infty}\left\{\frac{1}{T} \int_{-T / 2}^{T / 2} A(z, t) \exp (i \omega t) d t\right\} .
$$

Separating the field into CW and stochastic components, as in (5), we have

$$
S_{t}(\omega, L)=\left\langle|a(\omega, L)|^{2}\right\rangle+\left|A_{0}(L)\right|^{2} \delta(\omega)
$$

where \langle\rangle denotes ensemble average, and normalization by the sample interval. $\delta(\omega)$ is the Kronecker delta function.The noise term has zero mean, so $\langle a(\omega, z)\rangle=0$ and the cross 
terms vanish. It is convenient to remove the $\mathrm{CW}$ contribution from the power spectrum, so we define

$$
S(\omega, L)=S_{t}(\omega, L)+\left|A_{0}(L)\right|^{2} \delta(\omega)=\left\langle|a(\omega, L)|^{2}\right\rangle .
$$

Using (17)

$$
S(\omega, L)=\left\langle\left|B_{11} a(\omega, 0)+B_{12} a^{*}(-\omega, 0)\right|^{2}\right\rangle \exp (-\alpha L) .
$$

Because $a(\omega, 0)$ is a random process, amplitudes at distinct frequencies are uncorrelated, so $\left\langle a(\omega, 0) a^{*}(-\omega, 0)\right\rangle=0$ and

$$
S(\omega, L)=\left\{\left|B_{11}\right|^{2} S(\omega, 0)+\left|B_{12}\right|^{2} S(-\omega, 0)\right\} e^{-\alpha L}
$$

where $S(\omega, 0)=\left\langle a(\omega, 0) a^{*}(\omega, 0)\right\rangle$ is the power spectrum of $\tilde{a}(t, 0)$, the input noise. To simplify the analysis, we assume that the input noise spectrum is symmetric around the carrier (e.g., white noise): $S(\omega, 0)=S(-\omega, 0)$. (21) becomes

$$
S(\omega, L)=\left\{\left|B_{11}\right|^{2}+\left|B_{12}\right|^{2}\right\} S(\omega, 0) e^{-\alpha L} .
$$

A linear system can be treated as a special case with nonlinear coefficient $\gamma=0$. In this case, $k=\beta \omega^{2}, r_{f}=$ $r_{b}=0,\left|B_{11}\right|=1, B_{12}=0$ and

$$
S_{L}(\omega, L)=S(\omega, 0) e^{-\alpha L} .
$$

Using $S_{L}$ as a normalization factor, so that the normalized optical gain, or optical noise amplification in the nonlinear system is

$$
S_{O G}(\omega, L)=\frac{S(\omega, L)}{S_{L}(\omega, L)}=\left|B_{11}\right|^{2}+\left|B_{12}\right|^{2} .
$$

Fig. 1 gives the normalized optical spectra versus fiber length in a single span system using dispersion shifted fibers (DSF) with positive dispersion [see Fig. 1(a)] and negative dispersion [see Fig. 1(b)]. Fiber parameter used to obtain Fig. 1 are loss coefficient $\alpha=0.22 \mathrm{~dB} / \mathrm{km}$, input signal optical power $P_{\text {in }}=13 \mathrm{dBm}$, nonlinear coefficient $\gamma=$ $2.07 \mathrm{~W}^{-1} \mathrm{~km}^{-1}$, and fiber dispersion $D=2 \mathrm{ps} / \mathrm{nm} / \mathrm{km}$ for Fig. 1a and $D=-2 \mathrm{ps} / \mathrm{nm} / \mathrm{km}$ for Fig. 1(b) with $D$ defined as $D=2 \pi c \beta / \lambda^{2}$. Fig. 2 shows the optical spectra for different value of fiber dispersions with the fiber length fixed at 100 km and $P_{\text {in }}=15 \mathrm{dBm}$. In both cases of Fig. 1 and Fig. 2, optical noise are amplified around the carrier. The difference is that in anomalous dispersion regime, optical spectrum have two peaks at each side of the carrier while in the normal dispersion regime, spectra are single peaked. The amplification of optical spectra near the carrier can be explained as the spectrum broadening of the carrier caused by the nonlinear phase modulation between the signal and the broadband ASE.

In the case of coherent optical transmission, the whole optical spectrum is moved to IF after beating with the local oscillator. The frequency components beyond the range of the baseband filter will then be removed and thus causes receiver power penalty. Therefore, the broadening of the signal optical spectrum is the major source of degradation in coherent optical transmission systems. For IMDD optical systems, on the other hand, the photo diode detects the total optical power without wavelength discrimination and relative intensity noise of the optical signal is the major source of degradation related to MI. In the rest of this paper, we will focus our attention on the effects of MI on IMDD systems.

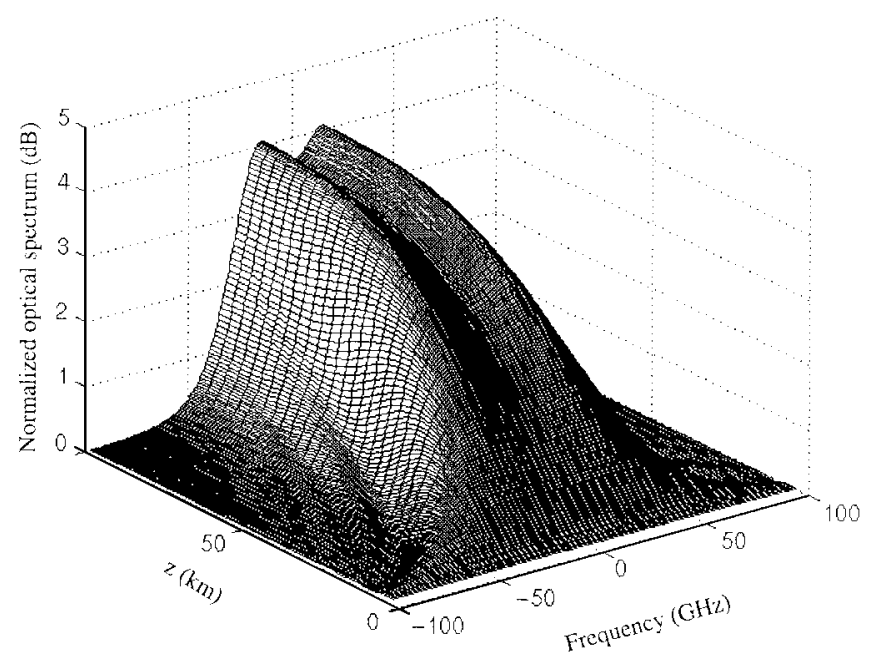

(a)

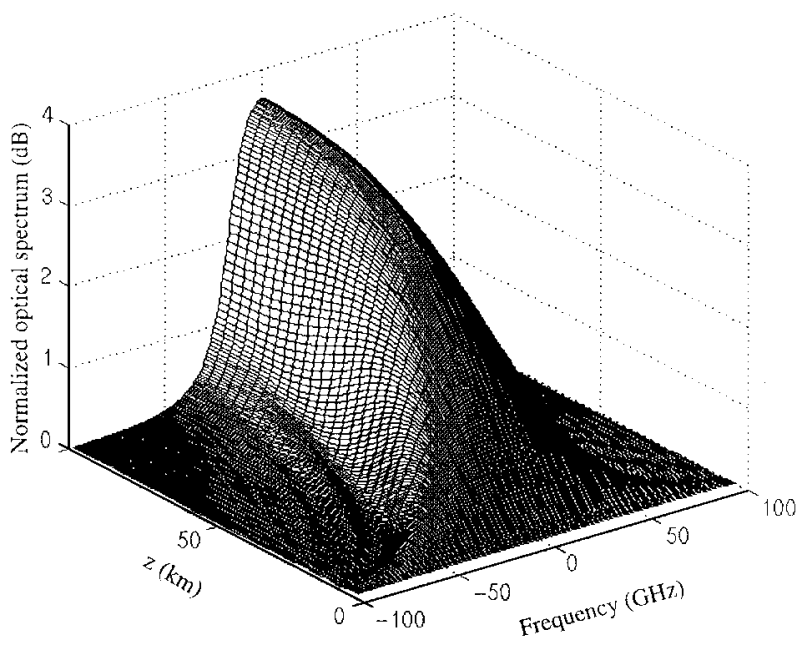

(b)

Fig. 1. Nonlinear amplification of ASE optical noise along the fiber longitudinal direction in a single span fiber system. Input optical signal power $P_{\text {in }}=13 \mathrm{dBm}$, fiber nonlinear coefficient $\gamma=2.07 \mathrm{~W}^{-1} \mathrm{~km}^{-1}$ and fiber loss $\alpha=0.22 \mathrm{~dB} / \mathrm{km}$. (a) Positive fiber dispersion $D=2 \mathrm{ps} / \mathrm{nm} / \mathrm{km}$. (b) Negative fiber dispersion $D=-2 \mathrm{ps} / \mathrm{nm} / \mathrm{km}$.

\section{Noise Description at a Direct-Detection Optical Receiver}

After the square-law detection of a photodiode, the photo current can be expressed as

$$
\begin{aligned}
I(t) & =\eta\left|A_{0}+\tilde{a}(t)\right|^{2} \\
& =\eta\left[\left|A_{0}\right|^{2}+A_{0} \tilde{a}^{*}(t, L)+A_{0}^{*} \tilde{a}(t, L)\right]
\end{aligned}
$$

where $\eta=\eta_{\text {in }} e / h \nu$ is the photodetection responsivity and $\eta_{\text {in }}$ includes quantum efficiency and coupling loss. Second order small terms have been omitted in the derivation of (25).

In an intensity-modulation and direct-detection (IMDD) system, the receiver performance is sensitive only to the amplitude noise of the photo current, which can be obtained from (25) as

$$
\delta I(t)=I(t)-I_{0}=\eta\left[A_{0}(L) \tilde{a}^{*}(t, L)+A_{0}^{*}(L) \tilde{a}(t, L)\right]
$$

where $I_{0}=\eta\left|A_{0}\right|^{2}$ is the photocurrent generated by the $\mathrm{CW}$ optical signal. 


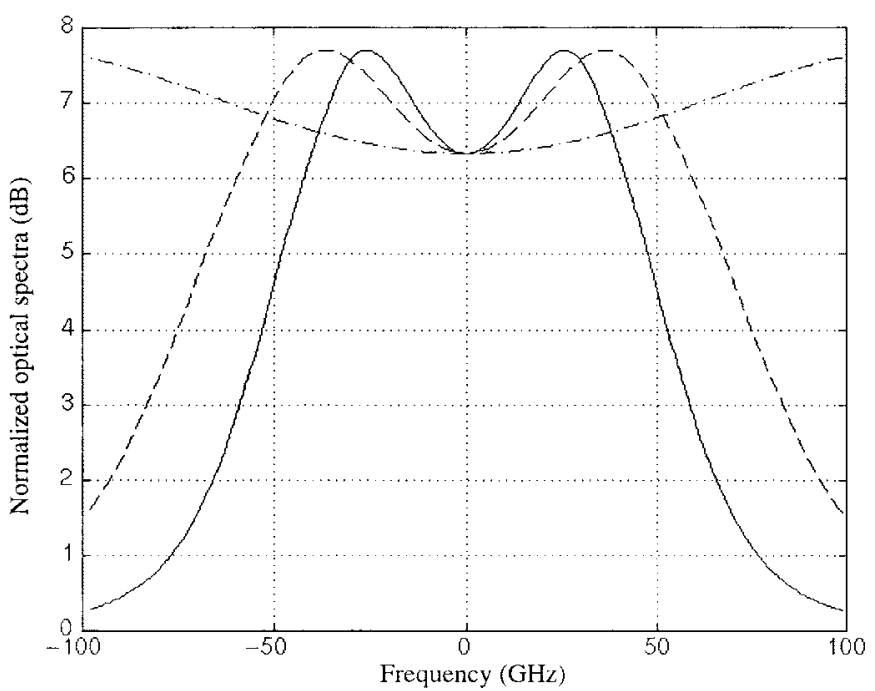

(a)

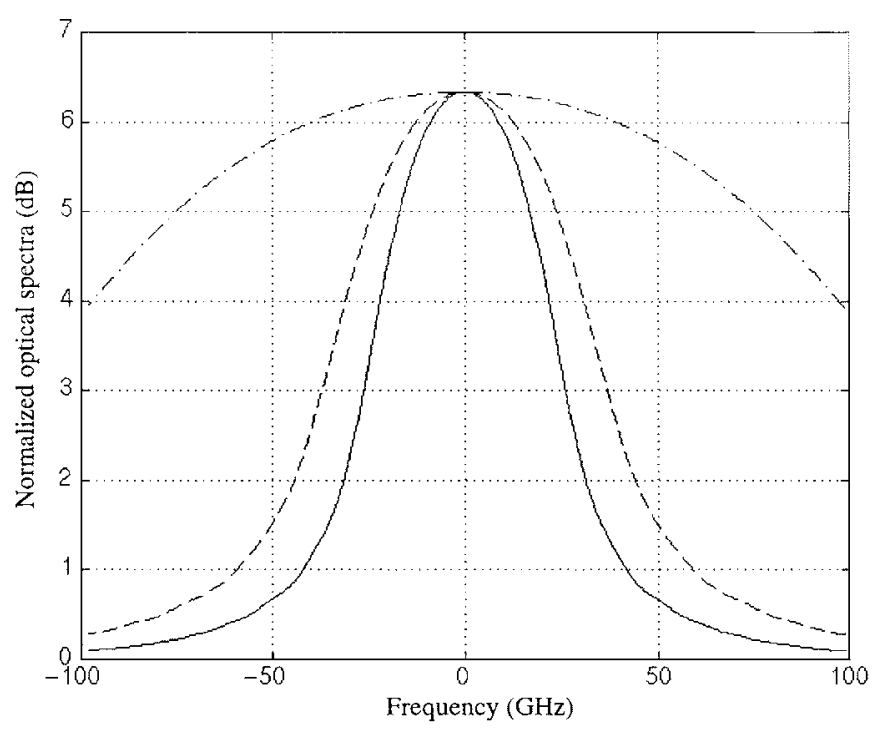

(b)

Fig. 2. Nonlinear amplification of ASE optical noise after $100 \mathrm{~km}$ transmission in a single span fiber system. Input optical signal power $P_{\text {in }}=15 \mathrm{dBm}$, fiber nonlinear coefficient $\gamma=2.07 \mathrm{~W}^{-1} \mathrm{~km}^{-1}$ and fiber loss $\alpha=0.22$ $\mathrm{dB} / \mathrm{km}$. (a) Positive fiber dispersion. Solid line: $D=1 \mathrm{ps} / \mathrm{nm} / \mathrm{km}$, dashed line: $D=0.5 \mathrm{ps} / \mathrm{nm} / \mathrm{km}$ and dash-dotted line: $D=0.05 \mathrm{ps} / \mathrm{nm} / \mathrm{km}$. (b) Negative fiber dispersion. Solid line: $D=-1 \mathrm{ps} / \mathrm{nm} / \mathrm{km}$, dashed line: $D=-0.5$ $\mathrm{ps} / \mathrm{nm} / \mathrm{km}$ and dash-dotted line: $D=-0.05 \mathrm{ps} / \mathrm{nm} / \mathrm{km}$.

The power spectrum of the noise photocurrent is the Fourier transformation of the autocorrelation of the time domain noise amplitude

$$
\begin{aligned}
& \rho_{n}(\omega)=\eta^{2}\left\{\left|A_{0}^{*} B_{11}+A_{0} B_{21}\right|^{2} S(\omega, 0)\right. \\
&\left.+\left|A_{0}^{*} B_{12}+A_{0} B_{22}\right|^{2} S(-\omega, 0)\right\} e^{-\alpha L}
\end{aligned}
$$

where $S(\omega, 0)$ is the power spectrum of $\tilde{a}(t, 0)$.

Under the same approximation as we used in the optical spectrum calculation, the input noise spectrum is symmetric around zero frequency $S(\omega, 0)=S(-\omega, 0),(27)$ becomes

$$
\begin{aligned}
\rho_{n}(\omega)= & \eta^{2}\left\{\left|B_{11}+B_{21}\right|^{2}+\left|B_{12}+B_{22}\right|^{2}\right\} \\
& \cdot\left|A_{0}(L)\right|^{2} S(\omega, 0) e^{-\alpha L} .
\end{aligned}
$$

Using (10)-(16), it is easy to prove that $\left|B_{11}+B_{21}\right|^{2}=$ $\left|B_{12}+B_{22}\right|^{2}$, therefore, (28) can be written as

$$
\rho_{n}(\omega)=2 P_{\mathrm{in}} \eta^{2}\left|B_{11}+B_{21}\right|^{2} S(\omega, 0) e^{-2 \alpha L}
$$

where $P_{\text {in }}$ is the input signal power such that $\left|A_{0}(L)\right|^{2}=$ $P_{\text {in }} \exp (-\alpha L)$.

Again, a linear system can be treated as a special case of a nonlinear system with the nonlinear coefficient $\gamma=0$. In this case, $k=\beta \omega^{2}, r_{f}=r_{b}=0,\left|B_{11}\right|=1$ and $B_{21}=0$. The electrical noise power spectrum in the linear system is then

$$
\rho_{0}(\omega)=2 P_{\text {in }} \eta^{2} S(\omega, 0) e^{-2 \alpha L} .
$$

Using $\rho_{0}(\omega)$ as the normalization factor, the normalized power spectrum of the receiver electrical noise, or normalized RIN, caused by fiber Kerr effect is therefore

$$
R(\omega)=\left|B_{11}+B_{21}\right|^{2} .
$$

Comparing (31) to (24), it is interesting to note that these two spectra are fundamentally different: relative phase difference between $B_{11}$ and $B_{21}$ has no impact in the optical noise amplification spectrum of (24) but it is important in the RIN spectrum as given in (31).

Fig. 3 gives the normalized RIN spectra versus fiber length in a single span system with anomalous dispersion [see Fig. 3(a)] and normal dispersion [see Fig. 3(b)]. Fiber parameter used in Fig. 3 are the same as those in Fig. 1. Fig. 4 shows the normalized RIN spectra for different value of fiber dispersions with the fiber length fixed at $100 \mathrm{~km}$ and $P_{\text {in }}=15 \mathrm{dBm}$. In the anomalous fiber dispersion regime [see Fig. 3(a)], two main side peaks of noise grow along $z$, the peak frequencies become closer to the carrier frequency and the widths become narrower in the process of propagating. On the other hand, if the fiber dispersion is in the normal regime, as shown in Fig. 3(b) and Fig. 4(b), noise power density becomes smaller than in the linear case in the vicinity of signal frequency. This implies a noise squeezing and system performance improvement. In either regime, the system performance is sensitive to the baseband electrical filter bandwidth.

It is worth mention here that in [10], similar RIN spectra for a lossless fiber have been calculated. However, since the fiber loss was neglected, the calculated RIN spectra are qualitatively different from a real situation. For example, in the case of normal fiber dispersion, the normalized RIN spectra was always less than $0 \mathrm{~dB}$ in the mean-field approximation. Fig. 5 shows the normalized RIN spectra with normal fiber dispersion calculated by the transfer matrix method when the fiber was divided into 1,10 , and 20 sections. The results converge very quickly when the number of sections is larger than ten. Here, the section lengths were chosen exponentially increase along the fiber longitudinal direction, that is, inversely proportional to the local optical power in the optical fiber. Fig. 6 shows the maximum calculation error versus the number of sections in the case of $100 \mathrm{~km}$ normal dispersion fiber with 10,15 , and $20 \mathrm{dBm}$ optical powers. When the number of sections is larger than 30, the maximum error becomes less than $0.01 \mathrm{~dB}$ for the $20 \mathrm{dBm}$ optical power case. For results reported in this work, 50 sections were used. 


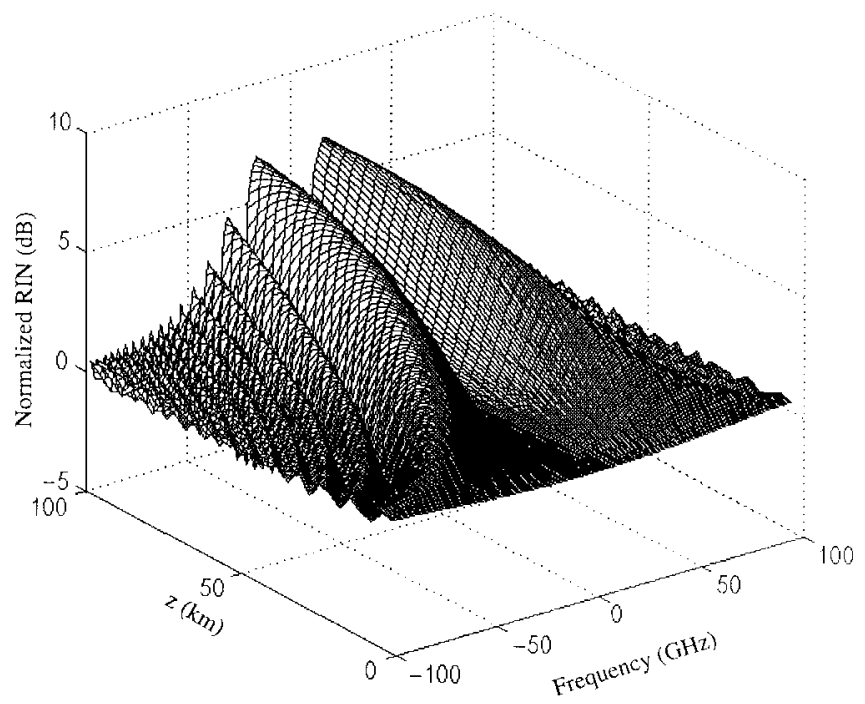

(a)

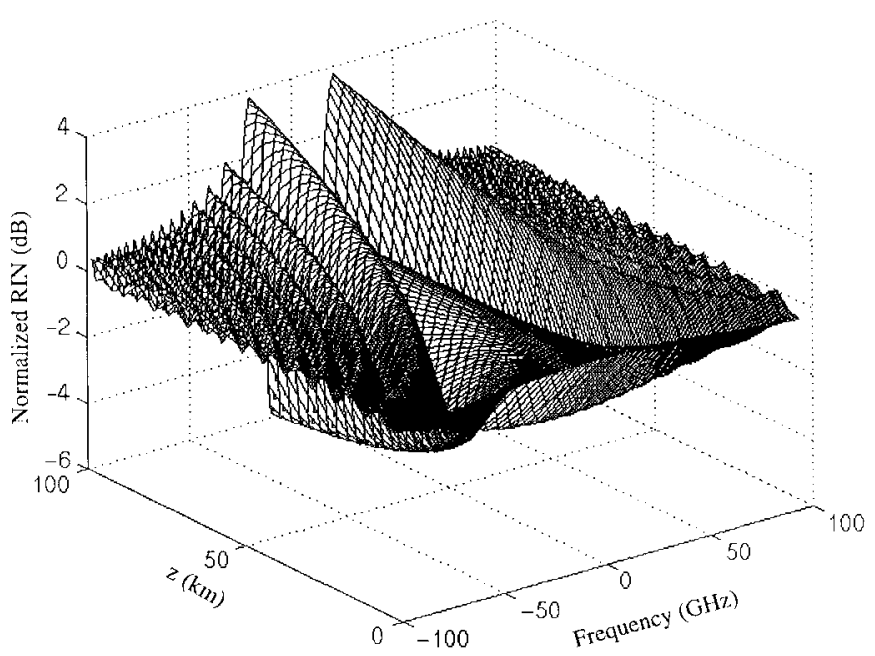

(b)

Fig. 3. Nonlinear amplification of relative intensity noise along the fiber longitudinal direction in a single span fiber system. Input optical signal power $P_{\text {in }}=13 \mathrm{dBm}$, fiber nonlinear coefficient $\gamma=2.07 \mathrm{~W}^{-1} \mathrm{~km}^{-1}$ and fiber loss $\alpha=0.22 \mathrm{~dB} / \mathrm{km}$. (a) Positive fiber dispersion $D=2 \mathrm{ps} / \mathrm{nm} / \mathrm{km}$. (b) Negative fiber dispersion $D=-2 \mathrm{ps} / \mathrm{nm} / \mathrm{km}$.

\section{Effect of Dispersion Compensation (DC)}

It is well known that DC is an important way to reduce the total system chromatic dispersion. We will show here that DC has also an important impact on the effect of MI. DC is usually accomplished by using dispersion compensating fibers or grating devices. Neglecting the nonlinear effect of the DC module, its transfer function can be represented by a conventional Jones matrix

$$
[C]=\left[\begin{array}{cc}
\exp [i \Phi(\omega)] & 0 \\
0 & \exp [-i \Phi(\omega)]
\end{array}\right]
$$

when the DC is made by a piece of optical fiber, $\Phi(\omega)=$ $\beta \omega^{2} z$.

The effect of DC on the optical system can be evaluated by simply multiplying the Jones matrix in (32) to the transfer matrix in (17). We can demonstrate that the RIN spectra at

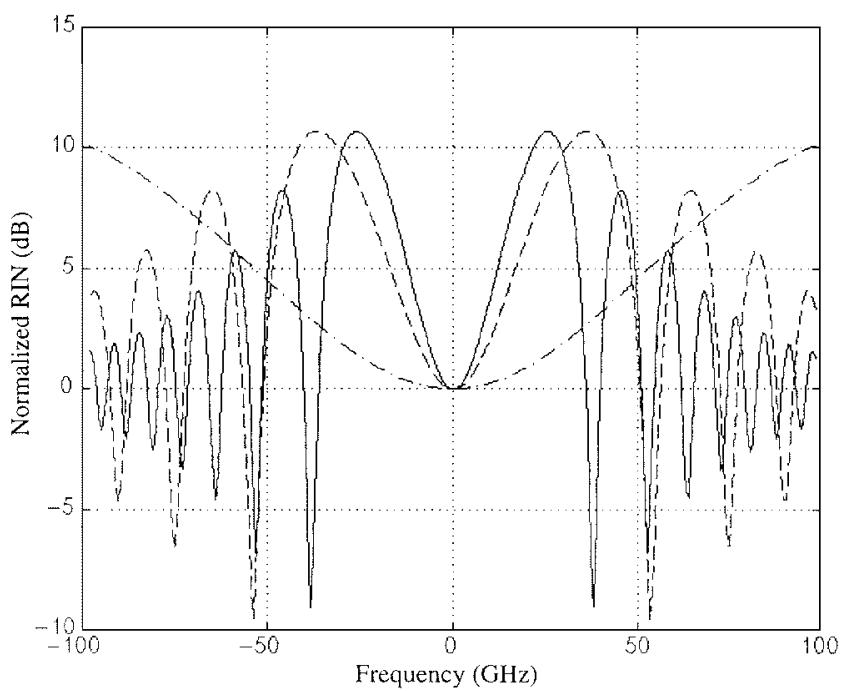

(a)

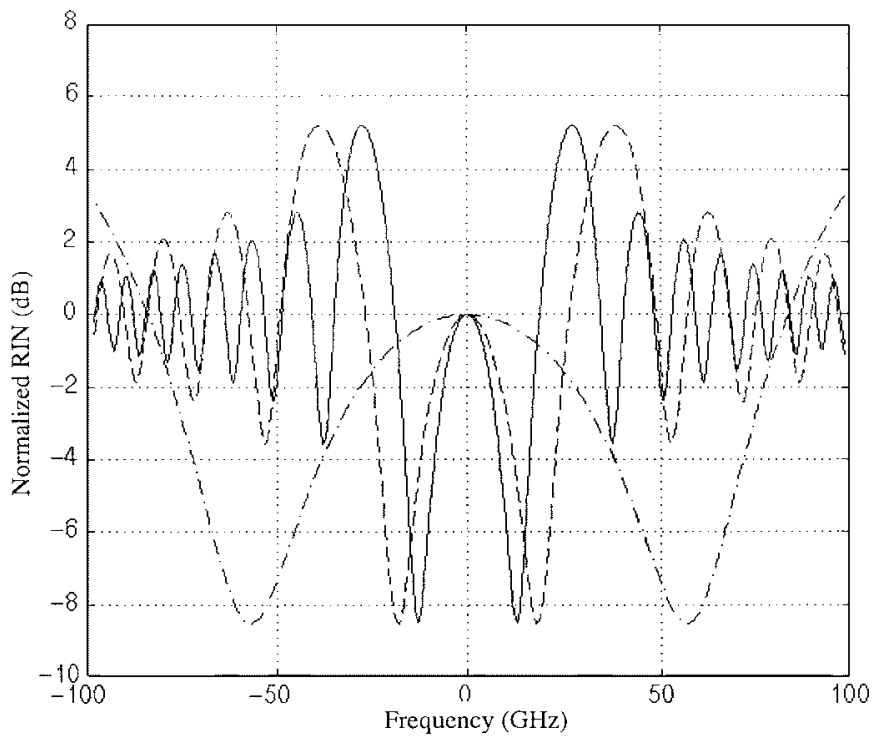

(b)

Fig. 4. Nonlinear amplification of relative intensity noise after $100 \mathrm{~km}$ transmission in a single span fiber system. Input optical signal power $P_{\mathrm{in}}=15 \mathrm{dBm}$, fiber nonlinear coefficient $\gamma=2.07 \mathrm{~W}^{-1} \mathrm{~km}^{-1}$ and fiber loss $\alpha=0.22 \mathrm{~dB} / \mathrm{km}$. (a) Positive fiber dispersion. Solid line: $D=1$ $\mathrm{ps} / \mathrm{nm} / \mathrm{km}$, dashed line: $D=0.5 \mathrm{ps} / \mathrm{nm} / \mathrm{km}$ and dash-dotted line: $D=0.05$ $\mathrm{ps} / \mathrm{nm} / \mathrm{km}$. (b) Negative fiber dispersion. Solid line: $D=-1 \mathrm{ps} / \mathrm{nm} / \mathrm{km}$, dashed line: $D=-0.5 \mathrm{ps} / \mathrm{nm} / \mathrm{km}$ and dash-dotted line: $D=-0.05$ $\mathrm{ps} / \mathrm{nm} / \mathrm{km}$.

the direct-detection optical receiver is sensitive not only to the value of DC but to the position of the DC module (DCM) as well. Let us take two examples to explain the reason. First, if the DCM is positioned after the nonlinear transmission fiber (at the receiver side), the combined transfer function becomes

$$
\begin{aligned}
{\left[\begin{array}{ll}
B_{11} & B_{12} \\
B_{21} & B_{22}
\end{array}\right] } & =\left[\begin{array}{cc}
\exp (i \Phi) & 0 \\
0 & \exp (-i \Phi)
\end{array}\right]\left[\begin{array}{ll}
B_{11}^{f} & B_{12}^{f} \\
B_{21}^{f} & B_{22}^{f}
\end{array}\right] \\
& =\left[\begin{array}{cc}
B_{11}^{f} \exp (i \Phi) & B_{12}^{f} \exp (i \Phi) \\
B_{21}^{f} \exp (-i \Phi) & B_{22}^{f} \exp (-i \Phi)
\end{array}\right] .
\end{aligned}
$$

The normalized RIN spectrum in (31) is then

$$
R(\omega)=\left|B_{11}^{f} \exp (i \Phi)+B_{21}^{f} \exp (-i \Phi)\right|^{2}
$$




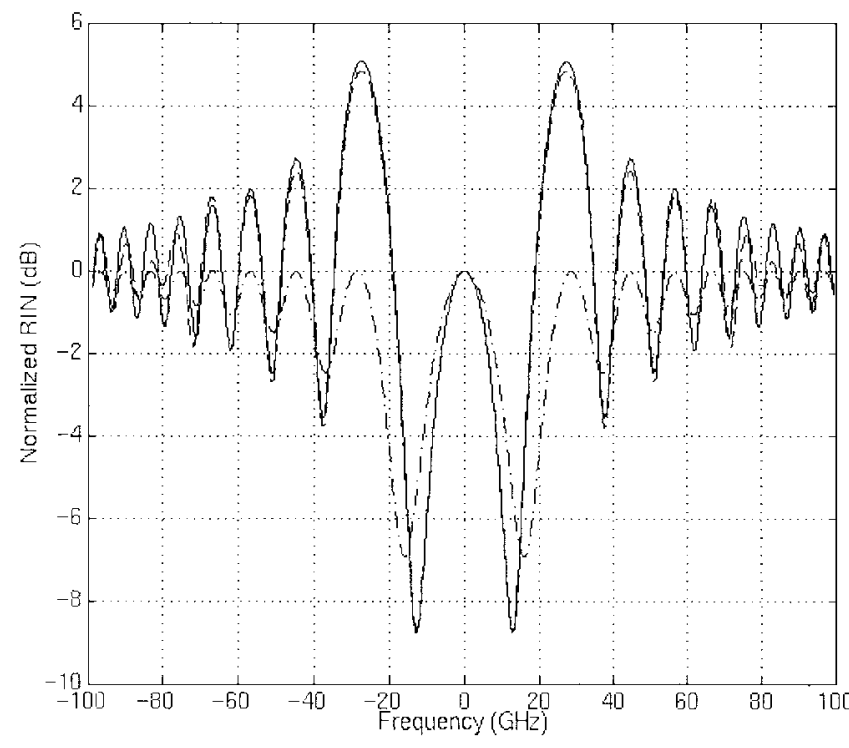

Fig. 5. Nonlinear amplification of relative intensity noise after $100 \mathrm{~km}$ transmission in a single span fiber system. Input optical signal power $P_{\text {in }}=15$ $\mathrm{dBm}$, fiber nonlinear coefficient $\gamma=2.07 \mathrm{~W}^{-1} \mathrm{~km}^{-1}$, fiber loss $\alpha=0.22$ $\mathrm{dB} / \mathrm{km}$ and $D=-1 \mathrm{ps} / \mathrm{nm} / \mathrm{km}$. dash-dotted line: $n=1$, dashed line: $n=10$ and solid line: $n=20$, where $n$ is the number of sections the fiber is divided into in the calculation.

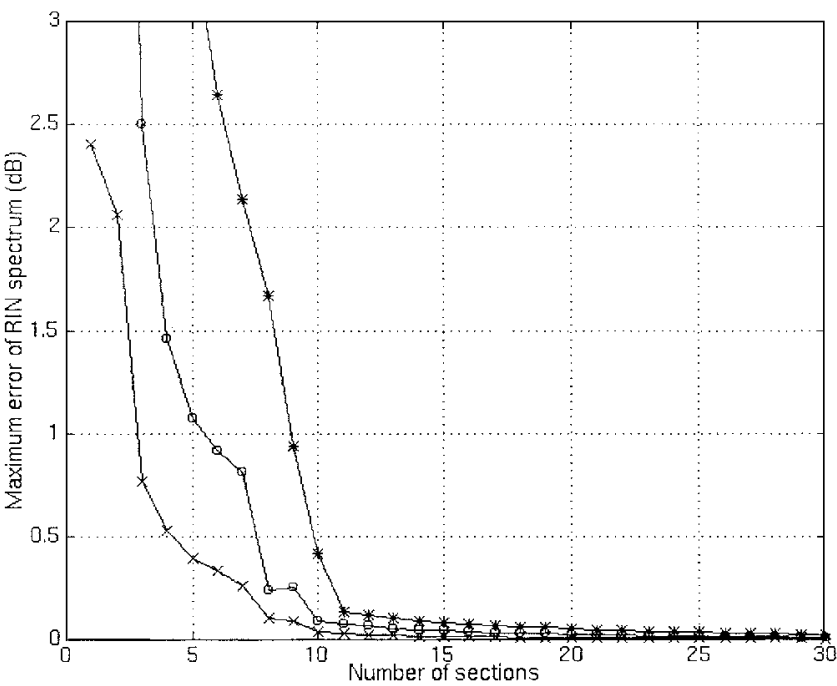

Fig. 6. Maximum error in the normalized RIN calculation versus the number of sections the fiber is divided into. Fiber length $L=100 \mathrm{~km}$, fiber nonlinear coefficient $\gamma=2.07 \mathrm{~W}^{-1} \mathrm{~km}^{-1}$, fiber loss $\alpha=0.22 \mathrm{~dB} / \mathrm{km}$ and $D=-1$ $\mathrm{ps} / \mathrm{nm} / \mathrm{km}$. Crosses: $P_{\mathrm{in}}=10 \mathrm{dBm}$, circles: $P_{\mathrm{in}}=15 \mathrm{dBm}$ and stars: $P_{\text {in }}=20 \mathrm{dBm}$.

where $B_{i j}^{f}(i=1,2, j=1,2)$ are the transfer function elements of the nonlinear transmission fiber only. Obviously, the normalized RIN spectrum is sensitive to the amount of DC. Fig. 7 shows an example of normalized RIN spectra without DC (solid line), with $50 \%$ of compensation (dash-dotted line) and with $100 \%$ compensation (dashed line). It is interesting to note here that $100 \%$ dispersion compensation does not necessarily bring the RIN spectrum to the linear case.

On the other hand, if the DCM is placed before the nonlinear transmission fiber (at the transmitter side), the total transfer

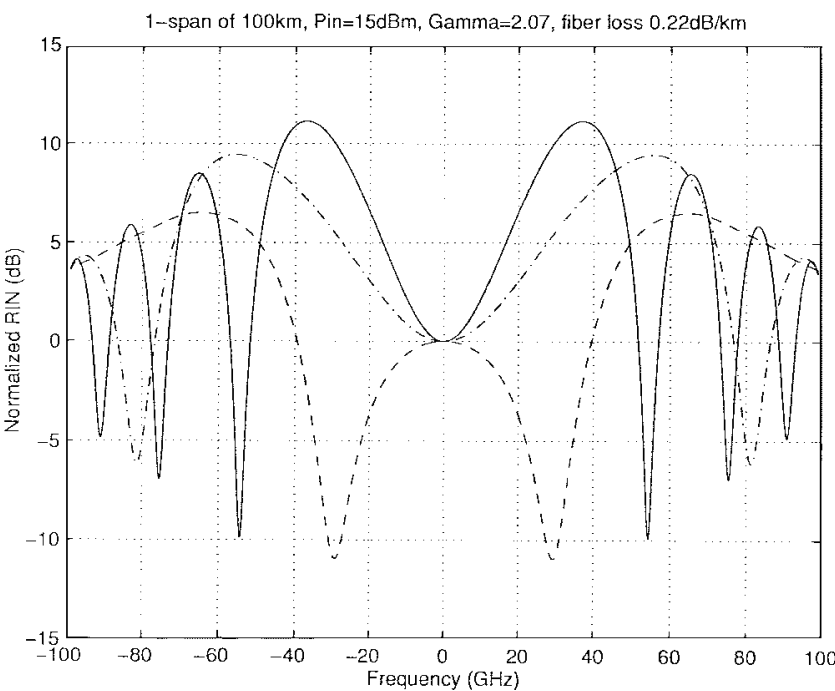

Fig. 7. Normalized RIN spectra for $0 \%$ (solid line), $50 \%$ (dash-dotted line) and $100 \%$ (dashed line) dispersion compensations. Fiber length $L=100 \mathrm{~km}$, fiber nonlinear coefficient $\gamma=2.07 \mathrm{~W}^{-1} \mathrm{~km}^{-1}$, fiber loss $\alpha=0.22 \mathrm{~dB} / \mathrm{km}$, $P_{\text {in }}=15 \mathrm{dBm}$ and $D=1 \mathrm{ps} / \mathrm{nm} / \mathrm{km}$.

matrix is

$$
\begin{aligned}
{\left[\begin{array}{ll}
B_{11} & B_{12} \\
B_{21} & B_{22}
\end{array}\right] } & =\left[\begin{array}{ll}
B_{11}^{f} & B_{12}^{f} \\
B_{21}^{f} & B_{22}^{f}
\end{array}\right]\left[\begin{array}{cc}
\exp (i \Phi) & 0 \\
0 & \exp (-i \Phi)
\end{array}\right] \\
& =\left[\begin{array}{ll}
B_{11}^{f} \exp (i \Phi) & B_{12}^{f} \exp (-i \Phi) \\
B_{21}^{f} \exp (i \Phi) & B_{22}^{f} \exp (-i \Phi)
\end{array}\right] .
\end{aligned}
$$

The normalized RIN spectrum in (31) then becomes

$$
R(\omega)=\left|B_{11}^{f} \exp (i \Phi)+B_{21}^{f} \exp (i \Phi)\right|^{2}=\left|B_{11}^{f}+B_{21}^{f}\right|^{2} .
$$

Therefore, dispersion compensation does not bring any difference into the normalized RIN spectrum if the DCM is placed at the transmitter side.

Another important observation is that the optical spectrum is not affected by the dispersion compensation, regardless of the position of the DCM. The reason of this can be found from (24) where the normalized optical spectrum is related to the absolute value of $B_{11}$ and $B_{12}$. It does not matter where the DCM is placed in the system, DC has no effect on the normalized optical spectrum.

\section{E. MI in Optical Amplified Multispan IMDD Fiber Systems}

In an optical fiber system of $N$ spans with $N$ EDFA's (1 post amplifier and $N-1$ line amplifiers) as schematically shown in Fig. 8, the span losses are compensated by the optical gain of the EDFA's. Suppose that all EDFA's have the same noise figure, the ASE noise power spectra generated by the $i$ th EDFA is

$$
S_{i}=h v\left(F G_{i}-1\right)
$$

here, the ASE spectrum is supposed to be white within the scale of receiver optical bandwidth. After the transmission through fibers, amplified by EDFA's and detected by the photo diode, the power spectrum of the detected RIN can be obtained by the multiplication of the transfer function of each span 


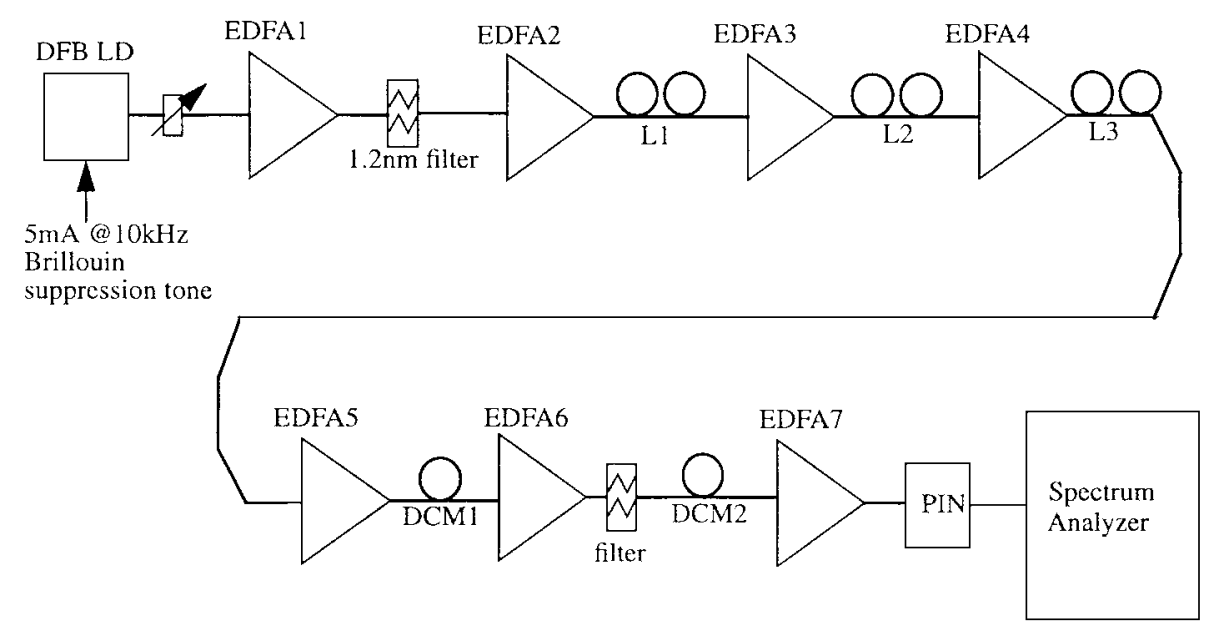

Fig. 8. Experimental setup. L1: $84.6 \mathrm{~km}(1307 \mathrm{ps} / \mathrm{nm})$, L2: $84.6 \mathrm{~km}(1291 \mathrm{ps} / \mathrm{nm}, \mathrm{L} 3: 90.4 \mathrm{~km}$ (1367 ps/nm). Optical powers at the output of EDFA5, EDFA6, and EDFA7 are less than $0 \mathrm{dBm}$.

of optical fiber, supposing that ASE generated by different EDFA's are uncorrelated

$$
\begin{aligned}
\rho(\omega)= & 2 h \nu P_{\text {in }} \eta^{2} \sum_{m=1}^{N}\left\{\left(F G_{N-m+1}-1\right) \mid B_{11}^{(N-m+1)}\right. \\
& \left.+\left.B_{21}^{(N-m+1)}\right|^{2}\right\}
\end{aligned}
$$

where $B_{i, j}^{(k)}$ are matrix elements defined as

$$
B^{(k)}=\prod_{m=1}^{k}\left[\begin{array}{ll}
B_{11} & B_{12} \\
B_{21} & B_{22}
\end{array}\right]_{m}
$$

and

$$
\left[\begin{array}{ll}
B_{11} & B_{12} \\
B_{21} & B_{22}
\end{array}\right]_{m}
$$

is the transfer function of the $m$ th fiber span.

Setting $\gamma=0$ to get the normalization factor, the normalized RIN spectrum is then:

$$
R(\omega)=\frac{\sum_{m=1}^{N}\left(F G_{N-m+1}-1\right)\left|B_{11}^{(N-m+1)}+B_{21}^{(N-m+1)}\right|^{2}}{\sum_{m=1}^{N}\left(F G_{N-m+1}-1\right)} .
$$

Assuming Gaussian statistics [11], the change of the standard deviation of the noise caused by fiber MI can be expressed in a simple way

$$
\delta \sigma=\frac{\sigma^{2}}{\sigma_{0}^{2}}=\int_{-\infty}^{\infty} R(\omega)|f(\omega)|^{2} d \omega
$$

where $\sigma$ and $\sigma_{0}$ are noise standard deviations in the nonlinear and linear cases, respectively and $f(\omega)$ is the receiver baseband filter transfer function.

In a direct-detection optical receiver with the effect of MI taken into account, the quality factor $Q$ can be expressed as (38) shown at the bottom of the page where $S$ is the signal level, $\sigma_{s h}, \sigma_{t h}, \sigma_{s p-s p}$ and $\sigma_{s-s p}$ are, respectively, the standard deviations of shot noise, thermal noise, spontaneousspontaneous beat noise and signal-spontaneous beat noise in the absence of MI. Subscripts 1 and 0 indicate the symbols for signal logical "1" and logical "0," respectively. MI affects mainly during signal logical "1" and the change in the RIN spectrum only affects the term representing signalspontaneous beat noise through the ratio $\delta \sigma_{1}$. However, considering that the signal extinction ratio is not infinite, signal power at logical " 0 " may also introduce MI through $\delta \sigma_{0}$, this effect should usually be very small. Optical spectrum change due to MI may introduce the $Q$ degradation through spontaneous-spontaneous beat noise, but it is expected to be the second-order small effect in the direct-detection optical receiver.

Equation (38) indicates that in the general case, system performance degradation due to MI depends on the proportionality of signal-spontaneous beat noise to other noises. Multispan optical amplified fiber systems, where signal-spontaneous beat noise predominates, are most sensitive to MI. Neglecting other noises, to first order, the system $Q$ degradation caused by the MI can be expressed in a very simple form as

$$
10 \log (\delta Q)=-5 \log (\delta \sigma)
$$

where $\delta Q$ is the ratio of $Q$ between nonlinear and linear cases.

$$
Q=\frac{S_{1}-S_{0}}{\sqrt{\sigma_{s h 1}^{2}+\sigma_{t h 1}^{2}+\sigma_{s p-s p 1}^{2}+\sigma_{s-s p 1}^{2} \delta \sigma_{1}}+\sqrt{\sigma_{s h 0}^{2}+\sigma_{t h 0}^{2}+\sigma_{s p-s p 0}^{2}+\sigma_{s-s p 0}^{2} \delta \sigma_{0}}}
$$




\section{EXPERIMENTAL SETUP}

The experimental configuration is shown in Fig. 8. The CW laser, emitting at $1543 \mathrm{~nm}$, has a sine wave tone superimposed on its drive current to dither the optical frequency so as to suppress Brillouin scattering. A separate experiment found that $5 \mathrm{~mA} \mathrm{p}$-p at $10 \mathrm{kHz}$ was adequate to ensure that Brillouin scattering was suppressed. It was found that the laser RIN could corrupt the results in some circumstance. When the drive current was $35 \mathrm{~mA}$ the RIN had a peak at $6 \mathrm{GHz}$ due to the laser relaxation oscillation $7 \mathrm{~dB}$ higher than the de level. With the insertion of $85 \mathrm{~km}$ of non dispersion shifted fiber (NDSF), this peak rose by a further $6 \mathrm{~dB}$ due to laser phase noise, which was converted to intensity noise by the fiber's dispersion. This converted noise was avoided by increasing the laser drive to $70 \mathrm{~mA}$, which moved the relaxation oscillation to higher frequency, and increased the damping rate to the relaxation oscillation thereby reducing the laser related RIN level.

In order to make sure that the measured receiver RIN is dominated by signal-spontaneous beat noise, we increased the broadband ASE noise from EDFA's intensionally. The role of the first optical amplifier in the link was to inject optical noise. It had a noise figure of $7.5 \mathrm{~dB}$ and the input optical power of $-32 \mathrm{dBm}$. The narrowband optical filter which followed kept the total ASE power less than that of the signal so as to decrease the spontaneous-spontaneous beat noise. The signal power was typically $0.5 \mathrm{~dB}$ less than the total power emerging from the EDFA, and this was taken into account setting the output of the line amplifiers. The first EDFA was the dominant source of ASE arriving at the p-i-n detector. The three line amplifiers had output power adjustable by computer control. The fiber spans all had loss coefficient measured by OTDR of $0.2 \mathrm{~dB} / \mathrm{km}$. The output power of the next two EDFA's was set below $0 \mathrm{dBm}$ to avoid nonlinear effects in the dispersion compensating fiber. The narrowband filter after EDFA suppressed ASE in $1560 \mathrm{~nm}$ region, which would have led to excessive spontaneous-spontaneous beat noise. The last optical amplifier was controlled to an output power of $4 \mathrm{dBm}$, just below the overload level of the p-i-n detector.

A calibration run was made, with an attenuator in place of the system. This measurement was substrated from all subsequent traces so as to take out the effect of the frequency response of the detection system. The calibration trace was $>20 \mathrm{~dB}$ higher than the noise floor for the whole $0-18 \mathrm{GHz}$ band.

\section{RESUlts AND Discussions}

The RIN spectra at the output of the three-span link are shown in Fig. 9 with line amplifier output power (signal power) controlled at $8,10,12$, and $14 \mathrm{dBm}$, for triangles, inverted triangles, squares, and circles, respectively. In Fig. 9, open points represent the measured spectra with the system configuration described in Fig. 8 except no DC was used. Continuous lines in the same figures are calculated using (31). Similarly, Fig. 10 shows the measured and calculated RIN spectra with the dispersion compensation of -4070

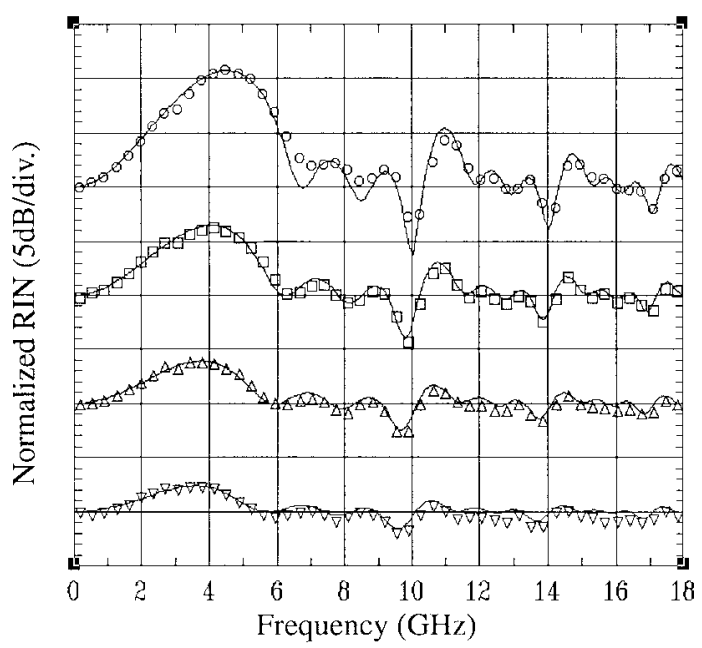

Fig. 9. Measured (open points) and calculated (solid lines) RIN spectra in the three-span NDSF system as described in Fig. 8 without dispersion compensation. The optical power at the output of EDFA2, EDFA3 and EDFA4 is $8 \mathrm{dBm}$ (triangles-down), $10 \mathrm{dBm}$ (triangles-up), $12 \mathrm{dBm}$ (squares) and $14 \mathrm{dBm}$ (circles). Curves are shifted for $10 \mathrm{~dB}$ between one and another for better display.

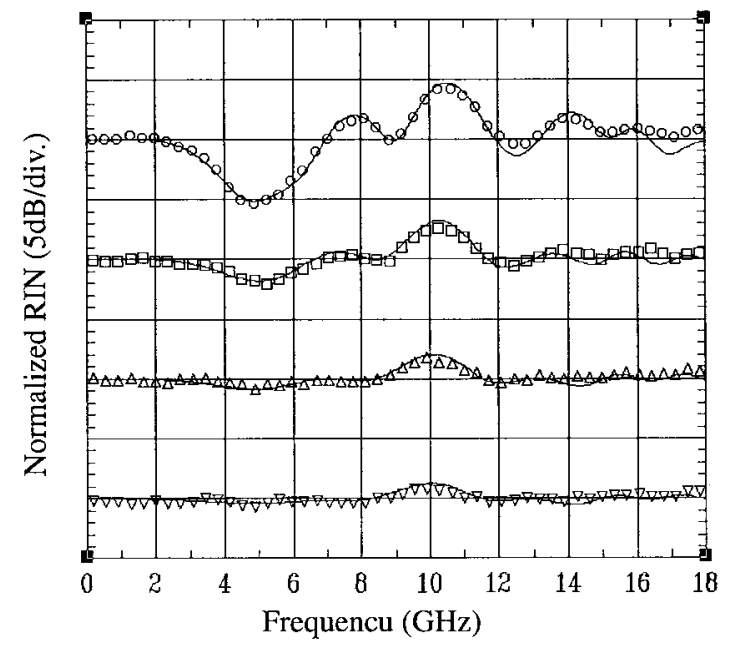

Fig. 10. Measured (open points) and calculated (solid lines) RIN spectra in the three-span NDSF system as described in Fig. 8 with $-4070 \mathrm{ps} / \mathrm{nm}$ dispersion compensation. The optical power at the output of EDFA2, EDFA3, and EDFA4 is $8 \mathrm{dBm}$ (triangles-down), $10 \mathrm{dBm}$ (triangles-up), $12 \mathrm{dBm}$ (squares), and $14 \mathrm{dBm}$ (circles). Curves are shifted for $10 \mathrm{~dB}$ between one and another for better display.

ps/nm at the receiver side as shown in Fig. 8. To obtain the theoretical results shown in Figs. 9 and 10, the fiber nonlinear coefficient used in the calculation was $\gamma=1.19 \mathrm{~W}^{-1} \mathrm{Km}^{-1}$ and other fiber parameters were chosen according to the values of NDSF used in the experiment as shown in Fig. 8. Very good agreement between measured and calculated results in the practical power range assures the validity of the two major approximations we have used in the transfer matrix formulation, namely, the linear approximation to the noise term and the insignificance of pump depletion.

Although the RIN spectra are independent of the signal data rate, the variance of the noise depends on the bandwidth of the baseband filter as explained in (37). In this paper, raised 


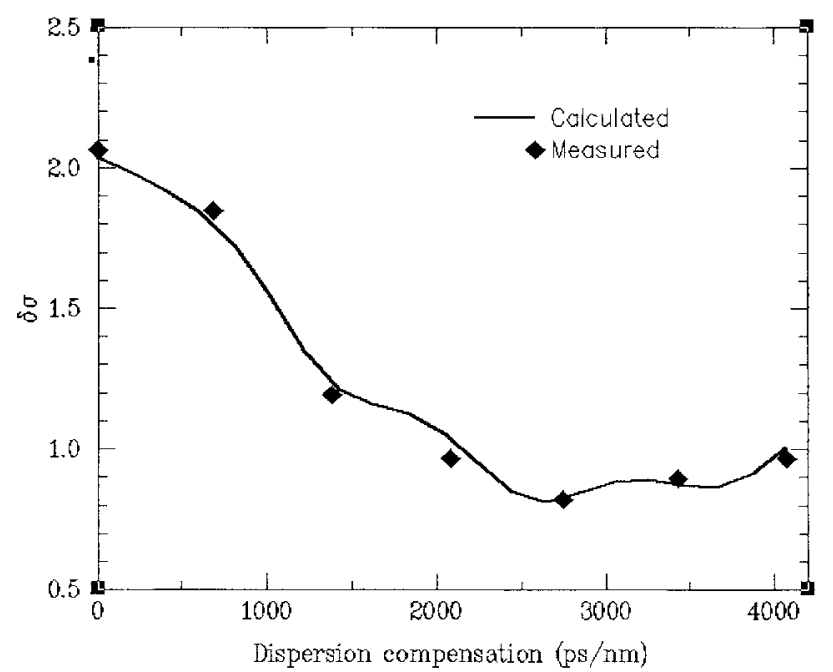

Fig. 11. Comparison of $\delta \sigma$ between calculation (solid line) and measurement (diamonds) for the three-span system described in Fig. 8 with optical power $P_{\text {in }}=12 \mathrm{dBm}$.

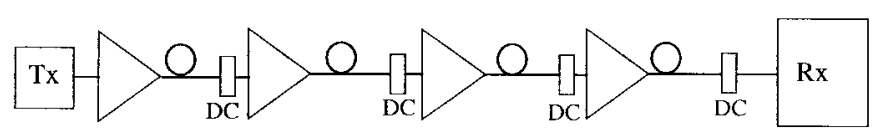

Distributed DC

(a)

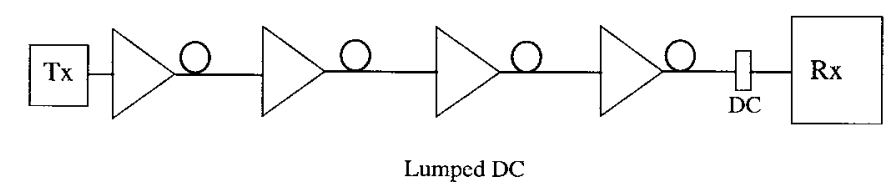

(b)

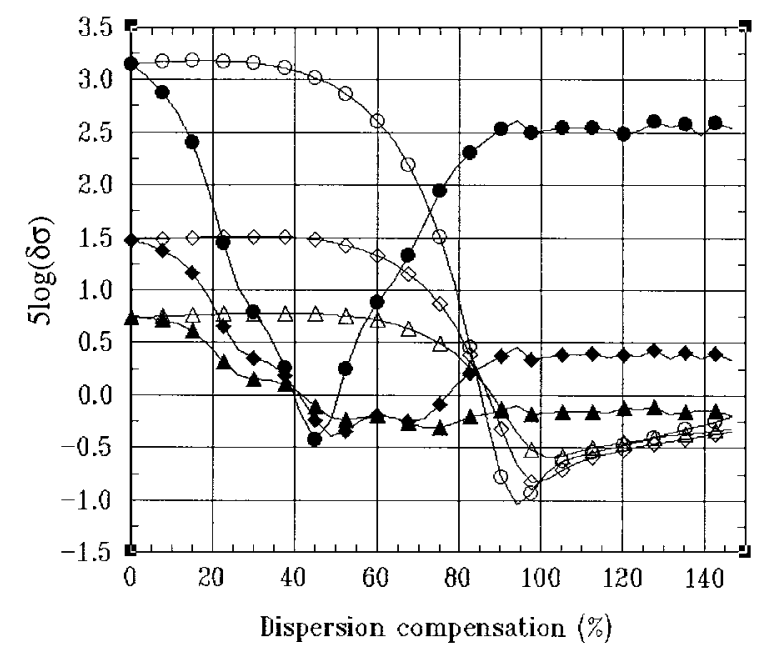

(c)

Fig. 12. Schematical illustration of optical systems with distributed DC (a) and a lumped DC in front of the receiver (b). (c) Comparison of $5 \log (\delta \sigma)$ versus the percentage of dispersion compensation between distributed DC (open points) and lumped DC (solid points) in the four-span NDSF systems shown in (a). Triangles: $P_{\mathrm{in}}=10 \mathrm{dBm}$, diamonds: $P_{\mathrm{in}}=12 \mathrm{dBm}$, and circles: $P_{\text {in }}=14 \mathrm{dBm}$.

cosine baseband filters are used in the system performance evaluation.

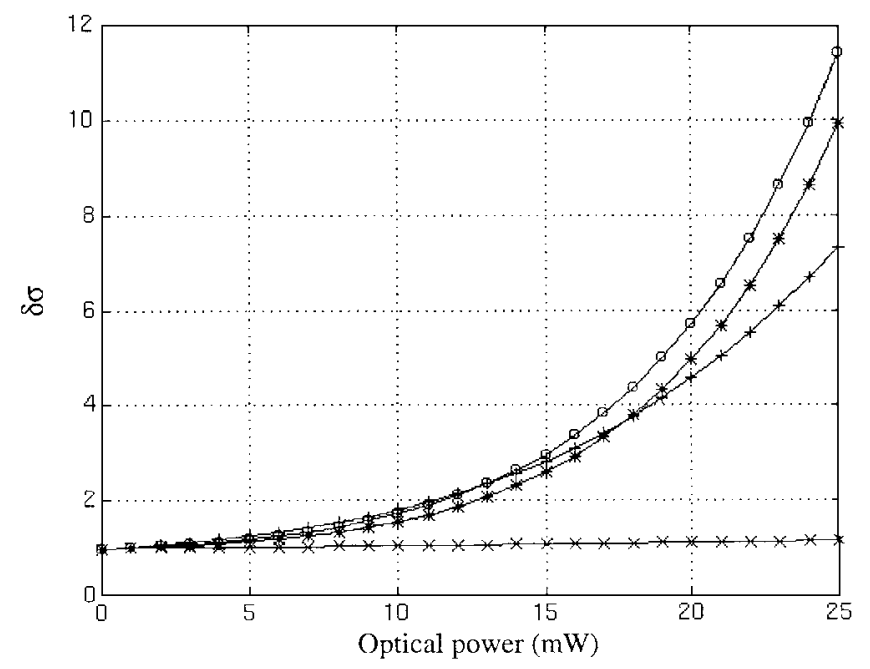

(a)

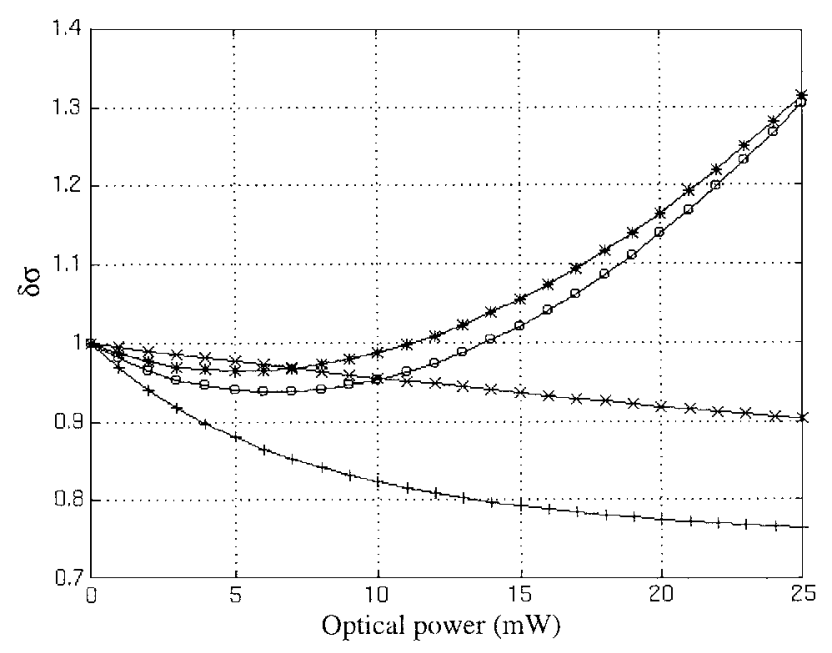

(b)

Fig. 13. $\delta \sigma$ versus optical power for a four-span DSF system with 100 $\mathrm{km}$ per span with fiber dispersion $+2 \mathrm{ps} / \mathrm{km} / \mathrm{nm}$ (a) and $-2 \mathrm{ps} / \mathrm{km} / \mathrm{nm}$ (b). Baseband filter bandwidth are $2 \mathrm{GHz}$ (crosses), $8 \mathrm{GHz}$ (pluses), $20 \mathrm{GHz}$ (stars), and $30 \mathrm{GHz}$ (circles).

Fig. 11 shows the effect of dispersion compensation on the ratio of noise standard deviation between nonlinear and linear cases for the three span fiber system described in Fig. 8. The optical power was $12 \mathrm{dBm}$ at the input of each fiber span and the filter bandwidth is $8 \mathrm{GHz}$. Both theoretical and experimental results demonstrate that $\delta \sigma$ approaches to its minimum when the DC is approximately $70 \%$ of the total system dispersion. Generally, the optimum level of dispersion compensation depends on the number of spans, electrical filter bandwidth, optical power levels and the dispersion in each fiber span. It is interesting to compare systems with distributed DC and a DC in front of the receiver, as schematically described in Fig. 12(a) and (b), for a four-span case with nondispersion shifted fibers (NDSF). Fig. 12(c) shows $5 \log (\delta \sigma)$, which is proportional to the system $Q$ degradation by MI, versus the percentage of dispersion compensation for these two kinds of DC configurations for three different optical power levels. In Fig. 12(c), the percentage of dispersion 


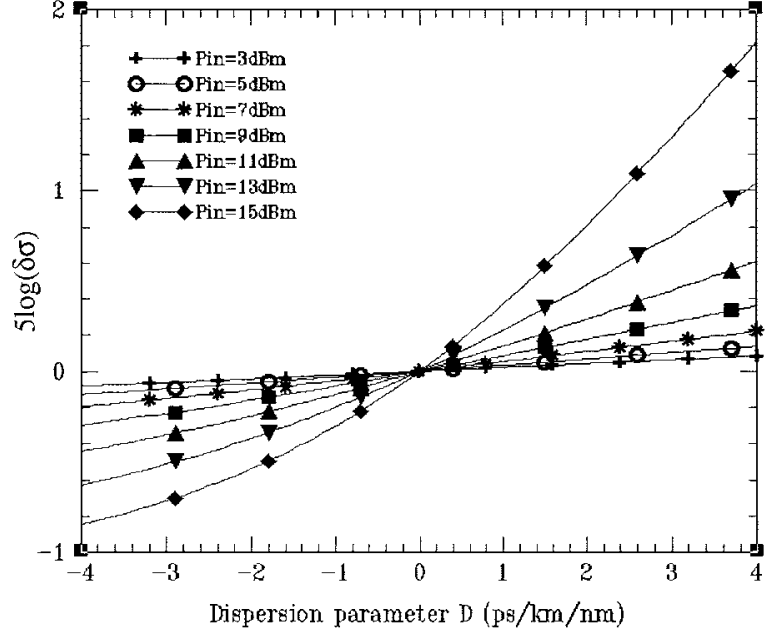

(a)

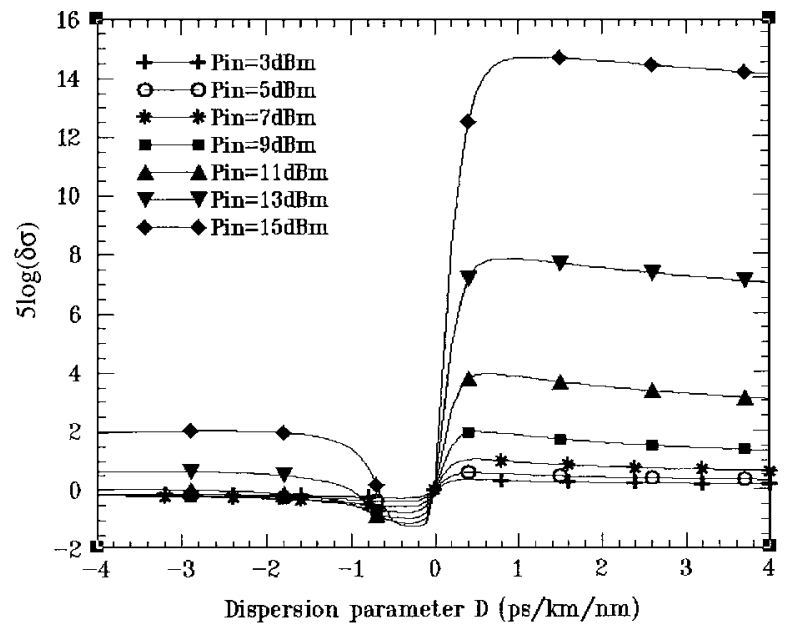

(c)

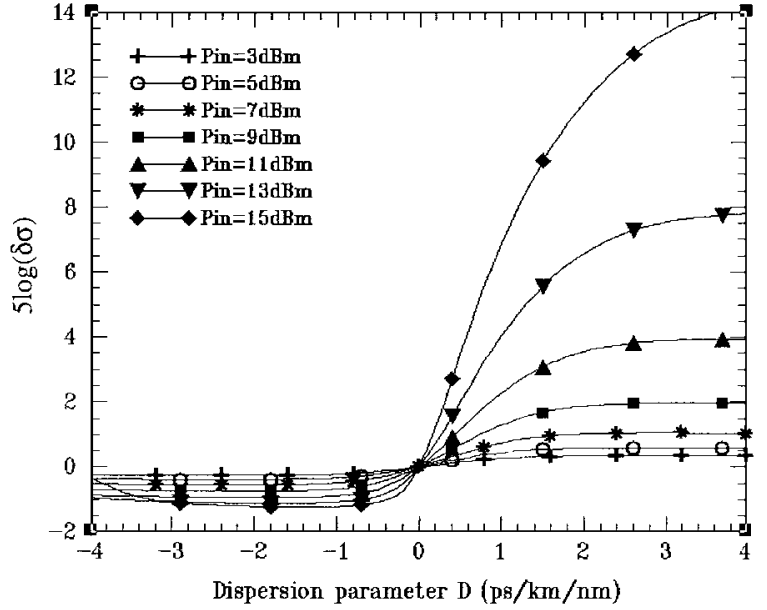

(b)

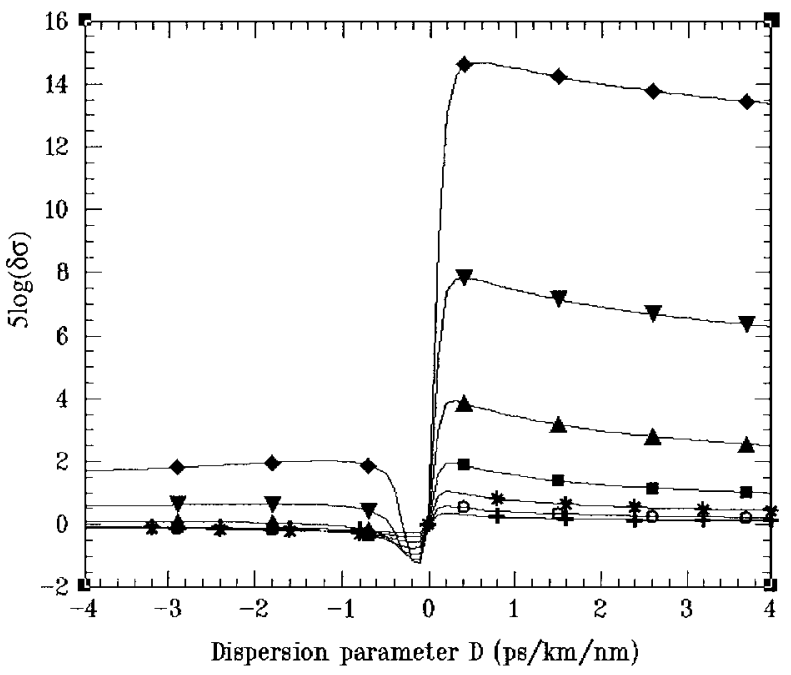

(d)

Fig. 14. A $5 \log (\delta \sigma)$ versus fiber dispersion for different signal power levels in a four-span system with $100 \mathrm{~km}$ per span. Baseband filter bandwidth are $2 \mathrm{GHz}$ (a), $8 \mathrm{GHz}$ (b), $20 \mathrm{GHz}$ (c), and $30 \mathrm{GHz}$ (d).

compensation is the total DC divided by total transmission fiber dispersion. The dispersion value used in the transmission fiber is $17 \mathrm{ps} / \mathrm{nm} / \mathrm{km}$ and the nonlinear effect in the DC fiber is neglected supposing the optical power in the DC fiber is small enough. The results demonstrate that the efficiency of DC, in terms of MI, is approximately $100 \%$ higher in the lumped DC configuration than in the distributed DC configuration. Even though the best compensation performance is obtained with distributed DC, but higher DC value usually implies higher loss (governed by the figure of merit of the DCM) and thus more ASE noise generated from EDFA's, therefore, lumped DC at the receiver may be a better choice in the system with low to moderate optical power levels.

In all above calculations and the corresponding experiments, NDSF's were used. In order to make a quantitative evaluation of the system with DSF's, Fig. 13(a) and (b) show the ratio of standard deviation between nonlinear and linear cases for $D=+2 \mathrm{ps} / \mathrm{km} / \mathrm{nm}$ and $D=-2 \mathrm{ps} / \mathrm{km} / \mathrm{nm}$, respectively. Raised cosine filters are used in the calculation with four different bandwidths, among which $1.8 \mathrm{GHz}$ bandwidth corresponds to a $2.5 \mathrm{~Gb} / \mathrm{s}$ (OC-48) system, $8 \mathrm{GHz}$ for a 10 $\mathrm{Gb} / \mathrm{s}$ (OC-192) system and $30 \mathrm{GHz}$ for a $40 \mathrm{~Gb} / \mathrm{s}$ (OC768) system. It is interesting to note that in the anomalous fiber dispersion regime, system performance always becomes worse with increasing signal power. On the other hand, in the normal dispersion regime, system sensitivity can be improved by the nonlinear process for OC-48 and OC-192 systems. However, for systems with higher bit rate, sensitivity degradations are also possible in the normal dispersion regime with high input signal powers. This degradation is believed to be caused by the subsidiary peaks as shown in Figs. 3(b) and 4(b).

Fig. 14(a)-(d), respectively, predict the $Q$ degradation factor $5 \log (\delta \sigma)$, caused by MI, versus fiber dispersions with the electrical bandwidths of 2, 8, 20, and $30 \mathrm{GHz}$. It is easy to see also from these plots that at zero dispersion, system performance degradations are always zero regardless of optical signal powers. This agrees with results of early studies [9], [12] under the assumption of unlimited system optical bandwidth. 


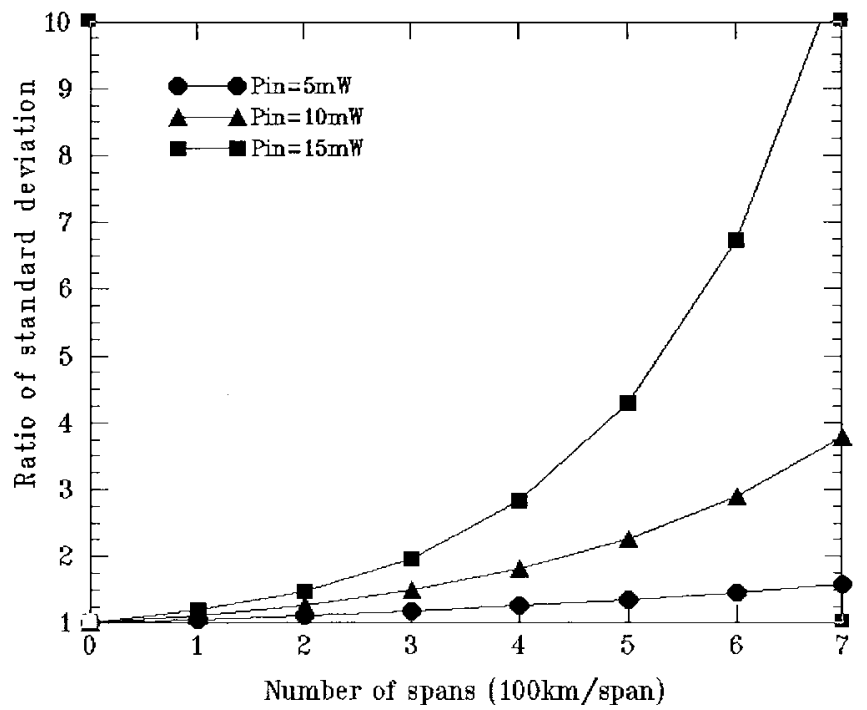

(a)

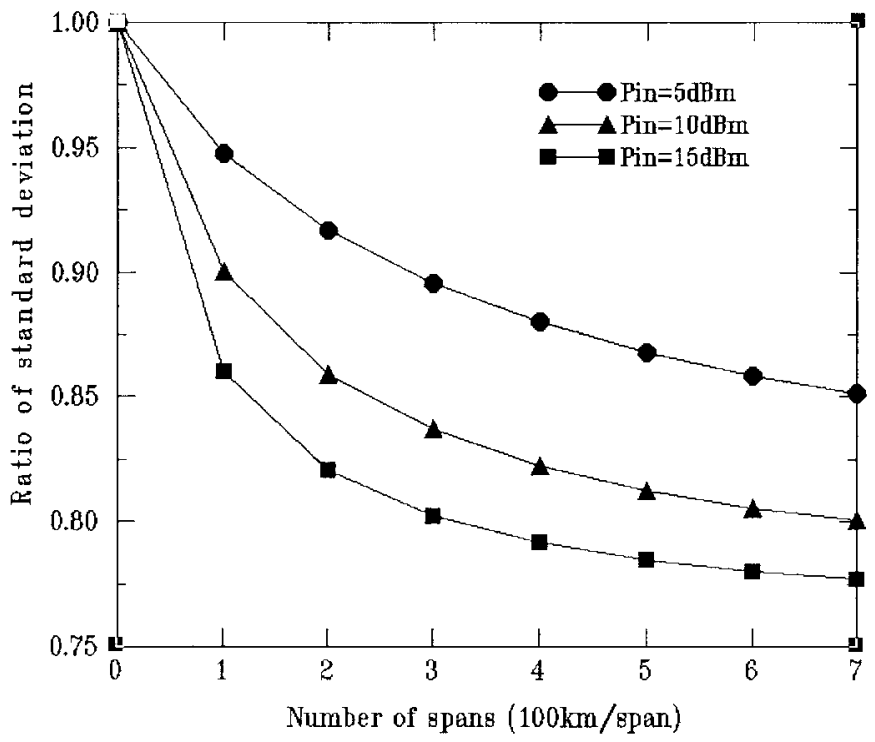

(b)

Fig. 15. $\delta \sigma$ versus number of fiber spans for different signal power levels Electrical bandwidth $8 \mathrm{GHz}, 100 \mathrm{~km}$ per span. Open circles: $D=-2$ $\mathrm{ps} / \mathrm{km} / \mathrm{nm}$ and $P_{\text {in }}=5 \mathrm{~mW}$, open triangles: $D=-2 \mathrm{ps} / \mathrm{km} / \mathrm{nm}$ and $P_{\text {in }}=10 \mathrm{~mW}$, open squares: $D=-2 \mathrm{ps} / \mathrm{km} / \mathrm{nm}$ and $P_{\text {in }}=15 \mathrm{~mW}$, solid circles: $D=2 \mathrm{ps} / \mathrm{km} / \mathrm{nm}$ and $P_{\mathrm{in}}=5 \mathrm{~mW}$, solid triangles: $D=2 \mathrm{ps} / \mathrm{km} / \mathrm{nm}$ and $P_{\text {in }}=10 \mathrm{~mW}$, solid squares: $D=2 \mathrm{ps} / \mathrm{km} / \mathrm{nm}$ and $P_{\text {in }}=15 \mathrm{~mW}$.

However, in ultra long fiber optical systems operating at zero dispersion, the nonlinearly broadened optical spectrum may spread well beyond the system effective optical bandwidth, creating large penalties [4], [13]. The linear approximation fails in that extreme situation.

Performance degradations caused by modulation instability were also calculated versus the number of optical amplified spans for OC-192 systems as shown in Fig. 15. The implication of this figure is that for normal dispersive fiber systems, nonlinearity improves the system performance compared to its linear counterpart as the number of spans are increased. However, the opposite is true for fiber systems with anomalous dispersion.

\section{CONCLUSION}

In conclusion, a transfer matrix model has been developed to analyze the impact of nonlinearity on the ASE noise for multispan fiber systems operating at nonzero dispersion. Excellent agreement between theory and experiment assures that we have a good understanding of the mechanism of MI and its impact in the performance of IMDD optical transmission systems. Contrary to the conventional notion that nonlinearity is always an impairment to system performance, we show that for system operating at normal dispersion, nonlinearity reduces the deleterious impact of ASE noise when compared with linear propagation. This improvement is achieved over a range of signal power level and receiver bandwidth. However, in the anomalous dispersion regime, nonlinearity always degrades the system performance when compared with linear propagation. Transmission at zero dispersion shows the same noise performance as for the linear case, which agrees with previous works. Dispersion compensation is an effective way to reduce the effect of MI and the optimum position of DC is discussed. The DC efficiency is approximately $100 \%$ higher in the lumped DC configuration than the distributed DC configuration where dispersion is compensated for within each fiber span.

Quantitative agreement between the theoretical and the measured RIN spectra also suggests that it is an alternative way to estimate the fiber parameters such as nonlinear coefficient $\gamma$ and dispersion $D$.

\section{REFERENCES}

[1] G. P. Agrawal, Nonlinear Fiber Optics. New York: Academic, 1989.

[2] A. Chraplyvy, "Limitations on lightwave communications imposed by optical-fiber nonlinearities," J. Lightwave Technol., vol. 8, pp. 1548-1557, 1990.

[3] D. Marcus, A. R. Chraplyvy, and R. W. Tkach, "Effect of fiber nonlinearity on ling-distance transmission," J. Lightwave Technol., vol. 9, pp. 121-128, 1991.

[4] D. Marcus, "Single-channel operation in very long nonlinear fibers with optical amplifiers at zero dispersion," J. Lightwave Technol., vol. 9, pp. 356-361, 1991.

[5] N. Henmi, T. Saito, and S. Nakaya, "An arrangement of transmissionfiber dispersions for increasing the spacing between optical amplifiers in lumped repeater systems," IEEE Photon. Technol. Lett., Vol. 5, pp. 1337-1340, 1993.

[6] D. Simeonidou, G. Long, and D. J. Butler, "Effect of local fiber dispersion on the performance of long-distance transmission systems with erbium-doped fiber amplifiers," in Proc. CLEO'95, Baltimore, MA, May 1995, CTul8

[7] J. P. Gordon and L. F. Mollenauer, "Phase noise in photonic communication systems using linear amplifiers," Opt. Lett., vol. 15, pp. 1351-1353, 1990.

[8] A. Mecozzi, "Error probability of amplified IMDD systems at zero dispersion," Electron. Lett., vol. 24, pp. 2136-2137, 1993.

[9] "Long-distance transmission at zero dispersion: Combined effect of the Kerr nonlinearity and the noise of the in-line amplifiers," J. Opt. Soc. Amer. B, vol. 12, pp. 462-465, 1994.

[10] M. Yu, G. P. Agrawal, and C. J. McKinstrie, "Pump-wave effects on the propagation of noisy signals in nonlinear dispersive media" J. Opt. Soc. Amer. B, vol. 13, pp. 1126-1132, 1995.

[11] D. Marcus, "Derivation of analytical expressions for the bit-error probability in lightwave systems with optical amplifiers," J. Lightwave Technol., vol. 8, pp 1816-1823, 1990.

[12] _ "Noise properties of four-wave mixing of signal and noise," Electron. Lett., vol. 30, pp. 1175-1177, 1994.

[13] E. Lichtman and S. G. Evangelides, "Reduction of the nonlinear impairment in ultralong lightwave systems by tailoring the fiber dispersion," Electron. Lett., vol. 30, pp. 346-348, 1994. 


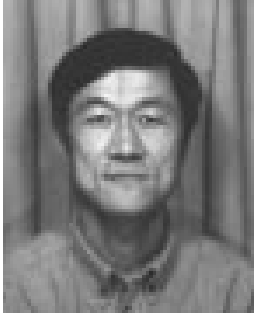

Rongqing Hui received the B.Sc. degree in microwave communications in 1982 and M.Sc. degree in lightwave technology in 1988, both from Beijing University of Posts and Telecommunications, Beijing, China. He received the Ph.D. degree in electronics engineering from Politecnico di Torino, Torino, Italy, in 1993.

From 1982 to 1985 , he taught at the Physics Department of Anhui University, Hefei, China, where he also conducted research into optical fibers and sensors. From 1985 to 1989, he was with the Optical Communication Laboratory of Beijing University of Posts and Telecommunications, where he worked in the field of coherent optical fiber communication systems and components. From 1989 to 1990, he held a Research Fellowship from Fundazione Ugo Bordoni, Rome, Italy, where he worked on nonlinear effects and optical injection locking of semiconductor laser devices. From 1990 to 1993, he was with the Department of Electronics, Politecnico di Torino, where he worked on optical communications and single frequency semiconductor laser devices. He also held a Fellowship from Italian Telecommunication Research Center (known as CSELT), Torino, Italy, during this period. From 1993 to 1994, he spent one year as a Postdoctoral Research Fellow at the University of Ottawa, Ottawa, Canada, where he worked on optical network architecture. He joined Bell-Northern Research (now part of Nortel), Ottawa, Ont., Canada, in 1994 as a Member of Scientific Staff, where he worked on high-speed optical transport networks. As the author or coauthor, he has published more than 40 technique papers in leading engineering journals in addition to numerous papers presented in international conferences. He also acted as the technique reviewer for various IEEE, IEE, and OSA journals.
Maurice O'Sullivan received the degree in physics (nonlinear spectroscopy) from the University of Toronto, Toronto, Ont., Canada.

He joined Northern Telecom in 1985, where he developed optical fiber characterization methods for manufacturing and designed optical fibers which were implemented using MCVD. He is presently a Senior Advisor to Advanced Transport Development at Nortel and leads an optical systems analysis group which studies propagation in multiwavelength high bit rate bidirectional optical systems.

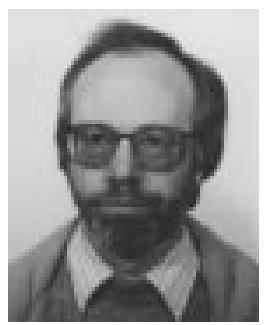

Alan Robinson received the B.A. degree in physics from the University of Cambridge, Cambridge, England, in 1973, followed by postgraduate work on metal fatigue in BCC metals at the Cavendish Laboratory in Cambridge.

In 1977, he joined STC's Standard Telecommunication Laboratories in Harlow, England (now part of Nortel Technology). He has worked on fiber manufacturing by plasma deposition, development of large core multimode and other specialist fibers, and investigating nuclear radiation effects in fibers. His other studies included multimode couplers, free-space optical interconnects, and fiber-Bragg gratings. He has been responsible for development of erbiumdoped fibers for amplifiers and is currently investigating propagation and nonlinear effects in WDM systems.

Mike Taylor, photograph and biography not available at the time of publication. 